\title{
Particle production as a function of multiplicity in pp collisions with ALICE
}

\author{
Cristiane Jahnke on behalf of the ALICE Collaboration ${ }^{* \dagger}$ \\ Universidade de São Paulo \\ E-mail: cristiane.jahnke@cern.ch
}

The study of particle production in high-multiplicity pp collisions has revealed unexpected collective effects. In order to investigate these features, particle yields as a function of event activity are measured by ALICE. The interplay between soft and hard processes is studied by measuring both heavy- and light-flavour particle yields as a function of charged particle multiplicity. In this work we present selected ALICE results of self normalised yield as a function of self normalised charged-particle multiplicity, measured in pp collisions.

7th Annual Conference on Large Hadron Collider Physics - LHCP2019

20-25 May, 2019

Puebla, Mexico

${ }^{*}$ Speaker.

${ }^{\dagger}$ Work supported by FAPESP. 


\section{Introduction}

High-multiplicity pp and $\mathrm{p}-\mathrm{Pb}$ collisions have shown features reminiscent of those observed in $\mathrm{Pb}-\mathrm{Pb}$ collisions, like azimuthal correlations and a mass-dependent hardening of transverse momentum $\left(p_{\mathrm{T}}\right)$ distributions $[1,2]$. Since high-multiplicity pp collisions reach similar number of charged particles as peripheral $\mathrm{Pb}-\mathrm{Pb}$ collisions, they can be used to investigate possible collective behaviour in small systems. Some studies show that multiple partonic interactions (MPI) can affect the particle production in such collisions, even for heavy-flavour hadrons [3, 4].

The ALICE detector $[5,6]$ consists of two separated spectrometers, one covering the midrapidity region $(|\eta|<0.9)$ and the other at forward-rapidity region $(-4<\eta<-2.5)$. The latter aims to detect muons, while the former measures electrons, hadrons, and photons.

ALICE has studied the interplay between soft and hard processes by analyzing particle production in both light-flavour ( $\mathrm{u}, \mathrm{d}, \mathrm{s}$ quarks) and heavy-flavour (c, b quarks) sectors. The event multiplicity is sensitive to low- $p_{\mathrm{T}}$ particles (soft) and is determined by the number of charged particles produced, while the hard process are assessed measuring high- $p_{\mathrm{T}}$ or heavy-flavour particles.

Studies of heavy-flavour hadron production as a function of charged-particle multiplicity were performed using B and D mesons and quarkonia (bound states of either $\mathrm{c}$ and $\overline{\mathrm{c}}$, or $\mathrm{b}$ and $\overline{\mathrm{b}}$ quarks). They can be measured in both mid- and forward-rapidity, using different decay channels (electrons or muons). Heavy-flavour quarks are mostly produced in initial hard scattering processes and they can form open ( $\mathrm{D}$ and $\mathrm{B}$ mesons) or hidden heavy-flavour particles (quarkonia). The production of $\mathrm{D}$ and $\mathrm{B}$ mesons can be used to test calculations based on perturbative Quantum Chromodynamics (pQCD). However, the production of quarkonia is not yet fully understood [7, 8, 9].

On the light-flavour sector, ALICE has studied charged and identified particles as a function of event multiplicity [10]. Particles produced at low transverse momenta $\left(p_{\mathrm{T}}<2 \mathrm{GeV} / c\right)$ are mainly from soft scattering processes, which are described by non-perturbative, phenomenological models.

In the ALICE experiment, the event multiplicity can be measured at mid- and forward-rapidity. The multiplicity at mid-rapidity is determined by the number of tracklets reconstructed in the Silicon Pixel Detector (SPD), which covers the pseudorapidity range $|\eta|<2$, consisting of the two innermost layers of the Inner Tracking System (ITS). At forward-rapidity, the multiplicity is determined by the V0 detector, a scintillator array, which is composed of two sections: V0A and V0C, located on either side of the interaction point. The V0A is located $340 \mathrm{~cm}$ away from the vertex and the V0C is located $90 \mathrm{~cm}$ away from the vertex.

\section{Results}

\subsection{Heavy-flavour hadron production as a function of charged-particle multiplicity}

In the ALICE detector, quarkonium production is measured via the dilepton decay channels. The central barrel detector (mid-rapidity) is used to measure $J / \psi$ in the dielectron decay channel while the muon spectrometer is used for the reconstruction of $J / \psi$ and $\Upsilon$ in the dimuon decay channel (forward-rapidity).

B- and D-meson production can be studied with ALICE via their hadronic or semi-leptonic decays. The hadronic and semi-electronic decays channel are studied at mid-rapidity, while the semi-muonic decay channel is studied at forward-rapidity. 
Figure 1 (left) shows the multiplicity dependence of inclusive $\mathrm{J} / \psi$ production at mid-rapidity in pp collisions at $\sqrt{s}=13 \mathrm{TeV}$ in different $p_{\mathrm{T}}$ intervals. Both particle yields and the average multiplicity are self normalised (divided by their average value from INEL $>0$ events, which are events with at least one SPD tracklet in $|\eta|<1$ ). Figure 1 (right) shows the production of electrons from heavy-flavour hadron decays as a function of charged-particle multiplicity in pp collisions at $\sqrt{s}=13 \mathrm{TeV}$. They are presented in several transverse momentum intervals. The results show a stronger than linear increase of the particle yields with multiplicity. Both results are compared with PYTHIA 8 [11], which includes MPI for the heavy-flavour production. PYTHIA 8 results describe qualitatively the momentum dependence observed for both open and hidden heavy-flavour production.
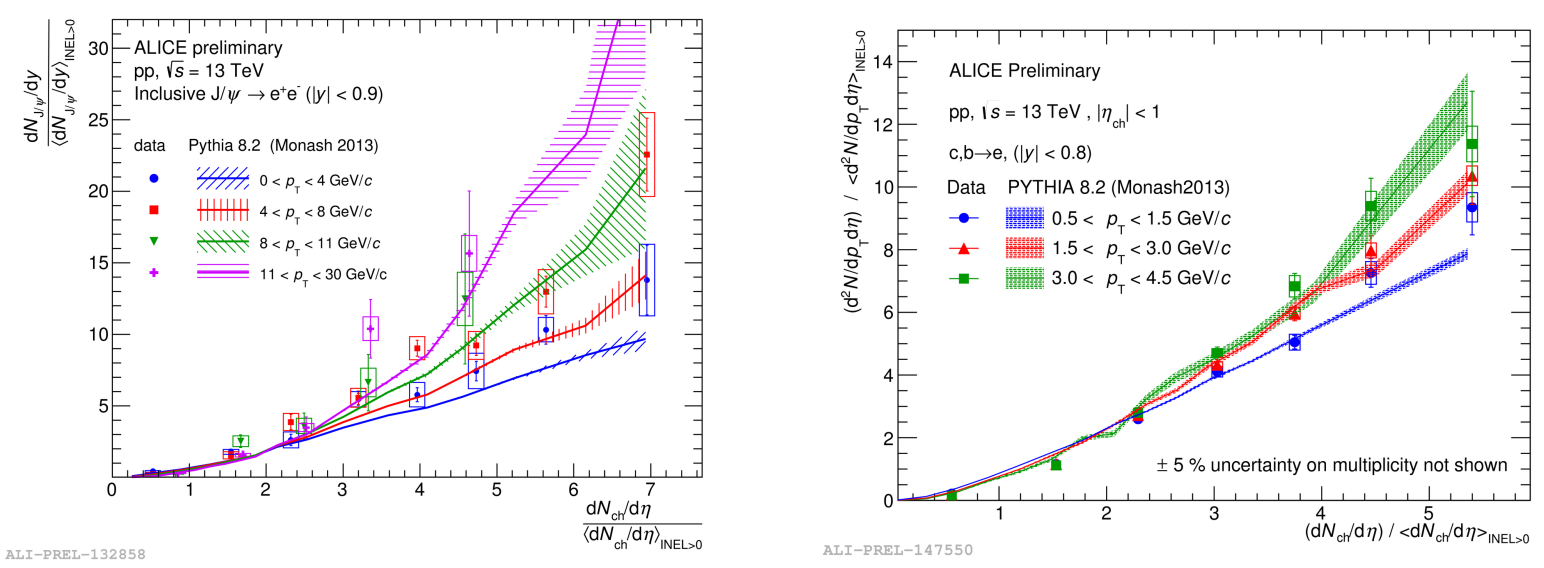

Figure 1: Multiplicity dependence of inclusive $\mathrm{J} / \psi$ production at mid-rapidity in pp collisions at $\sqrt{s}=$ $13 \mathrm{TeV}$ in different $p_{\mathrm{T}}$ intervals (left). Multiplicity dependence of inclusive electrons from heavy-flavour hadron decays production at mid-rapidity in pp collisions at $\sqrt{s}=13 \mathrm{TeV}$ in different $p_{\mathrm{T}}$ intervals (right). Both results are compared with PYTHIA 8 [11].

Figure 2 (left) shows the multiplicity dependence of inclusive $\mathrm{J} / \psi$ production compared with several theoretical models: PYTHIA 8 [11], EPOS 3 [12], Ferreiro et al. [13], and Kopeliovich et al. [14]. All models predict a stronger than linear increase of the $\mathrm{J} / \psi$ yield with respect to the event multiplicity, however, they fail to describe quantitatively the trend observed in data at very high multiplicity.

The results described above are measured as a function of charged-particle multiplicity, where the multiplicity was measured at mid-rapidity. Since the observables of interest (J/ $\psi$ or D and B mesons) are also measured at mid-rapidity, a correlation between them can have some influence in the results. This auto-correlation can be reduced if a rapidity gap is introduced between the observable and the event multiplicity measurement.

Figure 2 (right) shows the multiplicity dependence of inclusive $J / \psi$ and $\Upsilon$ production at forwardrapidity compared with the multiplicity dependence of inclusive $\mathrm{J} / \psi$ production at mid-rapidity in pp collisions at $\sqrt{s}=13 \mathrm{TeV}$. Forward-rapidity inclusive $\mathrm{J} / \psi$ and $\Upsilon$ production shows a linear increase with charged-particle multiplicity. In this case the event multiplicities were measured at mid-rapidity and possible auto-correlations should have a smaller impact. 

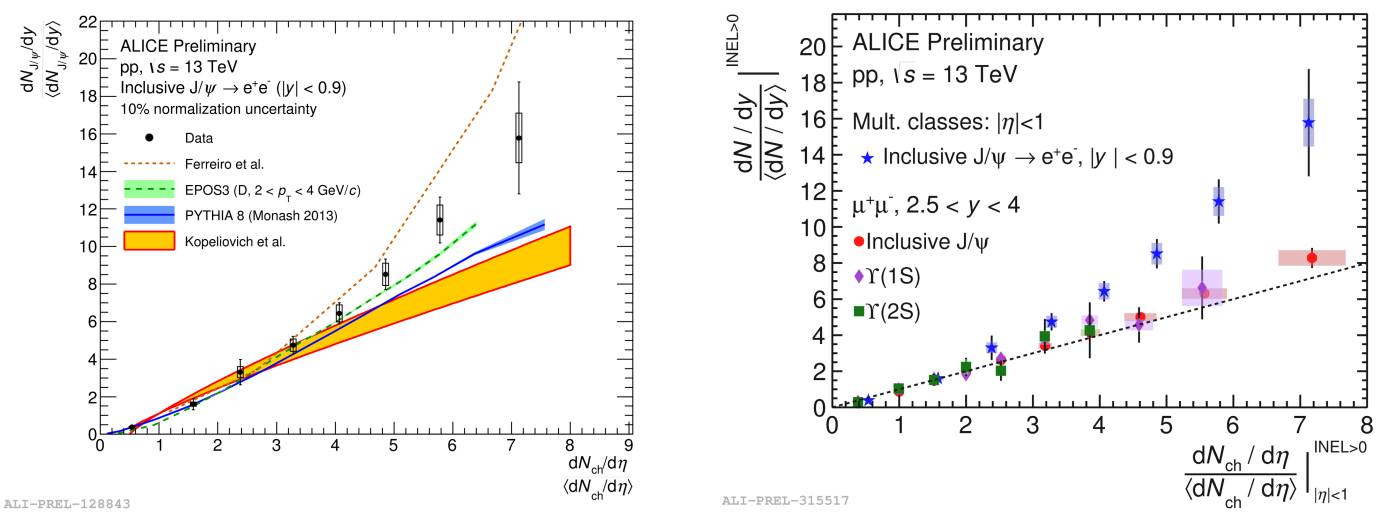

Figure 2: Multiplicity dependence of inclusive $J / \psi$ production at mid-rapidity in pp collisions at $\sqrt{s}=$ $13 \mathrm{TeV}$ compared with theoretical models (left). Multiplicity dependence of $\Upsilon$ production at forward-rapidity compared with multiplicity dependence of inclusive $\mathrm{J} / \psi$ production at mid-rapidity in pp collisions at $\sqrt{s}=$ $13 \mathrm{TeV}$ (right).

\subsection{Light-flavour hadron production as a function of charged-particle multiplicity}

Figure 3 (left) shows the multiplicity dependence of inclusive charged-particle production in pp collisions at $\sqrt{s}=13 \mathrm{TeV}$ and $\sqrt{s}=5 \mathrm{TeV}$. The light-flavour particles are measured at midrapidity $(|\eta|<0.8)$, while the event charged-particle multiplicities were measured at both mid- and forward-rapidities. The yields of charged particles with $4<p_{\mathrm{T}}<10 \mathrm{GeV} / c$ increase faster than linear with the charged-particle multiplicity and show a similar trend for both mid- and forwardrapidity estimators. This effect illustrates the correlation between multiplicity (low- $p_{\mathrm{T}}$ particles) and high- $p_{\mathrm{T}}$ particle production $[15,16]$. Figure 3 (right) shows self normalised yield of charged particles as a function of charged-particle multiplicity for several $p_{\mathrm{T}}$ intervals [17]. The results are described by the EPOS LHC model. This trend with $p_{\mathrm{T}}$ is in agreement with the heavy-flavour results presented in the previous section and it is the expected behaviour from jets [16].

Figure 4 (left) shows the $p_{\mathrm{T}}$-differential yields of strange particles measured in pp collisions at $\sqrt{s}=7 \mathrm{TeV}$ [18]. The results are presented for several multiplicity intervals and the $p_{\mathrm{T}}$ spectra become harder as the multiplicity increases. The hardening of the spectra is more pronounced for higher mass particles. Figure 4 (right) shows the $p_{\mathrm{T}}$-integrated yield ratios to pions $\left(\pi^{+}+\pi^{-}\right)$ as a function of charged-particle multiplicity. The results show an enhancement of strange to non-strange hadron production when increasing the charged-particle multiplicity $[18,19]$. The measurements are in agreement with results in $\mathrm{p}-\mathrm{Pb}$ collisions, which reach values similar to $\mathrm{Pb}-$ $\mathrm{Pb}$ peripheral collisions, indicating that a similar effect is present in all collision systems. 

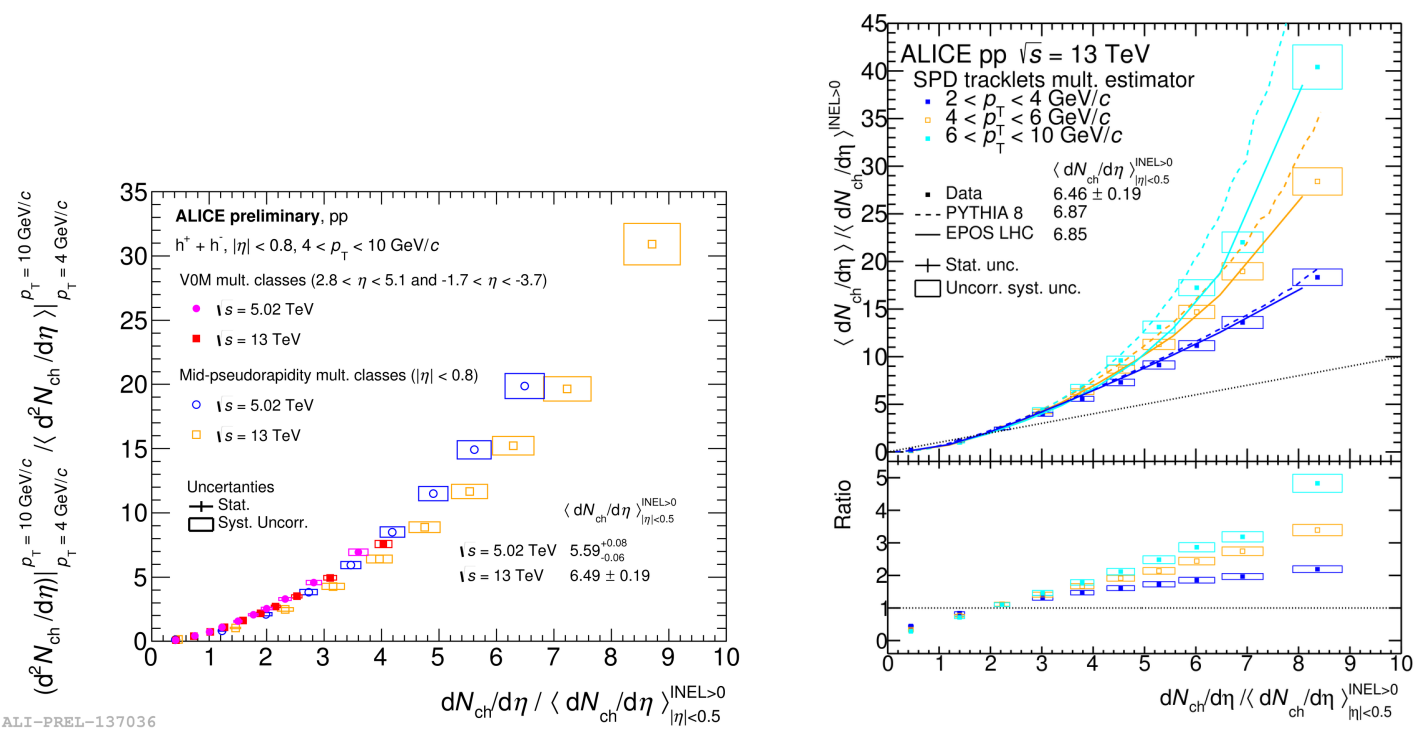

Figure 3: Multiplicity dependence of unidentified charged-particle production with $4<p_{\mathrm{T}}<10 \mathrm{GeV} / c$ in pp collisions at $\sqrt{s}=13 \mathrm{TeV}$ and $\sqrt{s}=5 \mathrm{TeV}$. The results are presented for two different multiplicity estimators, at mid-rapidity and at forward-rapidity (left). Multiplicity dependence of inclusive chargedparticle production in pp collisions at $\sqrt{s}=13 \mathrm{TeV}$ in different $p_{\mathrm{T}}$ intervals, for charged-particle multiplicity measured at mid-rapidity. The results are compared with PYTHIA 8 and EPOS LHC models [17] (right). 

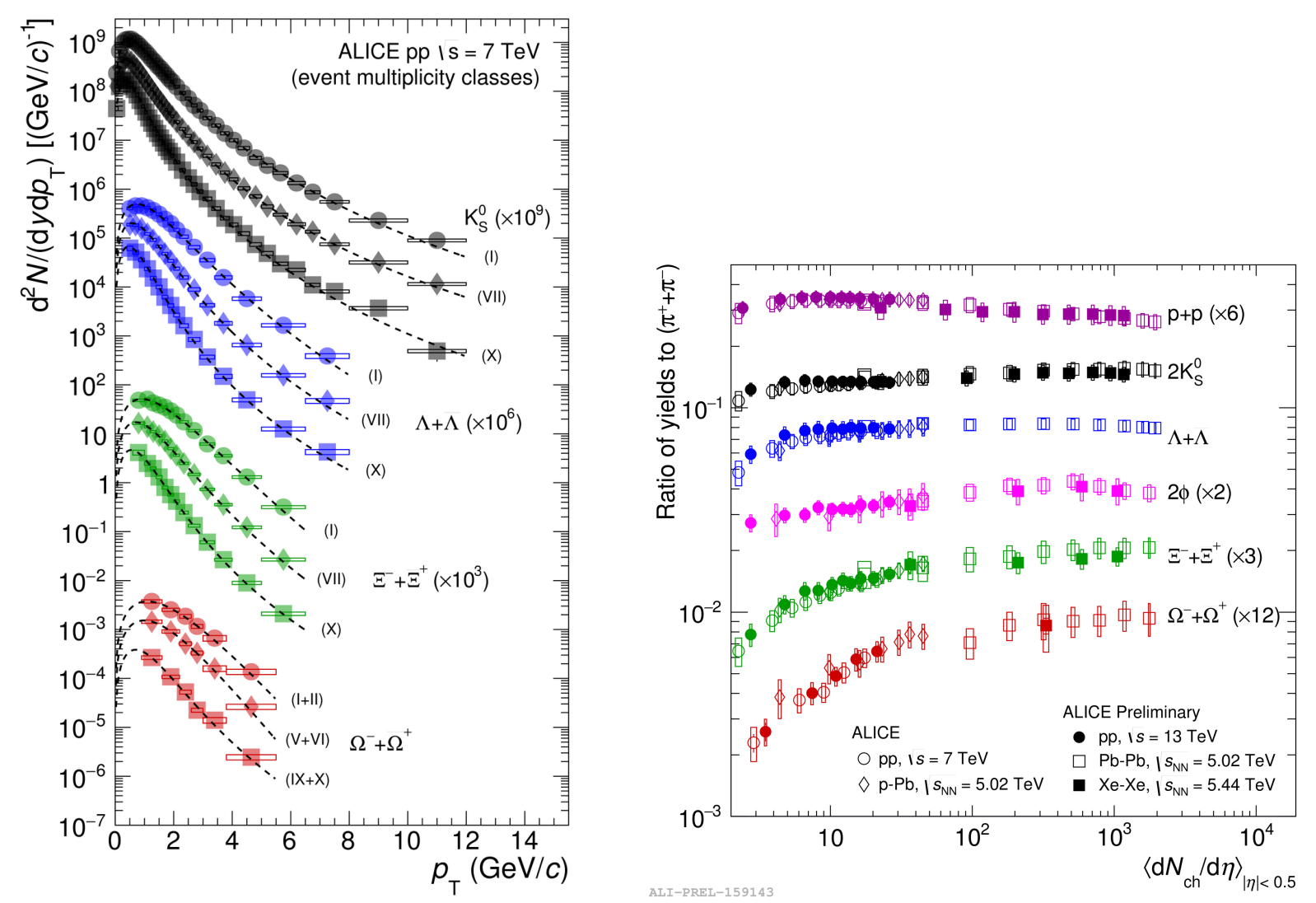

Figure 4: Transverse momentum differential yields of strange particles in pp collisions at $\sqrt{s}=7 \mathrm{TeV}$ [18] (left). Integrated transverse momentum yield ratios to pions $\left(\pi^{+}+\pi^{-}\right)$as a function of charged-particle multiplicity for $\mathrm{pp}, \mathrm{p}-\mathrm{Pb}, \mathrm{Pb}-\mathrm{Pb}$ and $\mathrm{Xe}-\mathrm{Xe}$ collisions $[18,19]$ (right). 


\section{Summary}

The measurements of self normalised yields as a function of charged-particle multiplicity show a stronger than linear increase for heavy-flavour particles measured at mid-rapidity. For quarkonia measurements at forward rapidity, a linear enhancement is observed. This can be due to the fact that there is a rapidity gap between the quarkonia measurement and the event multiplicity determination. However, a stronger than linear increase for charged-particle yields of $p_{\mathrm{T}}>4 \mathrm{GeV} / c$ is observed for both mid- and forward-rapidity multiplicity estimators. A stronger rise for higher $p_{\mathrm{T}}$ is observed for both heavy- and light-flavour particles measured at mid-rapidity. The enhancement of strange to non-strange hadron production is observed with increasing multiplicity and the strangeness production in high multiplicity pp events reaches values similar to those observed in heavy-ion collisions. This feature can be an indication of collectivity in high multiplicity pp collisions.

\section{References}

[1] CMS Collaboration, V. Khachatryan et al., "Observation of Long-Range Near-Side Angular Correlations in Proton-Proton Collisions at the LHC," JHEP 09 (2010) 091, arXiv: 1009.4122 [hep-ex].

[2] ALICE Collaboration, B. Abelev et al., "Long-range angular correlations on the near and away side in $p$-Pb collisions at $\sqrt{s_{N N}}=5.02$ TeV," Phys. Lett. B719 (2013) 29-41, arXiv: 1212.2001 [nucl-ex].

[3] ALICE Collaboration, B. Abelev et al., " $J / \psi$ Production as a Function of Charged Particle Multiplicity in pp Collisions at $\sqrt{s}=7 \mathrm{TeV}$," Phys. Lett. B712 (2012) 165-175, arXiv:1202.2816 [hep-ex].

[4] ALICE Collaboration, J. Adam et al., "Measurement of charm and beauty production at central rapidity versus charged-particle multiplicity in proton-proton collisions at $\sqrt{s}=7 \mathrm{TeV}$," JHEP 09 (2015) 148, arXiv:1505.00664 [nucl-ex].

[5] ALICE Collaboration, K. Aamodt et al., "The ALICE experiment at the CERN LHC," JINST 3 (2008) S08002.

[6] ALICE Collaboration, B. B. Abelev et al., "Performance of the ALICE Experiment at the CERN LHC," Int. J. Mod. Phys. A29 (2014) 1430044, arXiv:1402.4476 [nucl-ex].

[7] H. Fritzsch, "Producing Heavy Quark Flavors in Hadronic Collisions: A Test of Quantum Chromodynamics," Phys. Lett. 67B (1977) 217-221.

[8] A. Andronic et al., "Heavy-flavour and quarkonium production in the LHC era: from proton-proton to heavy-ion collisions," Eur. Phys. J. C76 no. 3, (2016) 107, arXiv:1506.03981 [nucl-ex].

[9] R. Baier and R. Ruckl, "Hadronic Production of $J / \psi$ and Upsilon: Transverse Momentum Distributions,” Phys. Lett. 102B (1981) 364-370.

[10] ALICE Collaboration, S. Acharya et al., "Multiplicity dependence of light-flavor hadron production in pp collisions at $\sqrt{s}=7$ TeV," Phys. Rev. C99 no. 2, (2019) 024906, arXiv: 1807.11321 [nucl-ex].

[11] T. Sjostrand, S. Mrenna, and P. Z. Skands, “A Brief Introduction to PYTHIA 8.1," Comput. Phys. Commun. 178 (2008) 852-867, arXiv:0710.3820 [hep-ph]. 
[12] H. J. Drescher, M. Hladik, S. Ostapchenko, T. Pierog, and K. Werner, "Parton based Gribov-Regge theory," Phys. Rept. 350 (2001) 93-289, arXiv: hep-ph/0007198 [hep-ph] .

[13] E. G. Ferreiro and C. Pajares, "High multiplicity $p p$ events and $J / \psi$ production at LHC," Phys. Rev. C86 (2012) 034903, arXiv:1203.5936 [hep-ph] .

[14] B. Z. Kopeliovich, H. J. Pirner, I. K. Potashnikova, K. Reygers, and I. Schmidt, “ $J / \psi$ in high-multiplicity pp collisions: Lessons from pA collisions,” Phys. Rev. D88 no. 11, (2013) 116002 , arXiv:1308.3638 [hep-ph].

[15] S. G. Weber, A. Dubla, A. Andronic, and A. Morsch, "Elucidating the multiplicity dependence of $J / \psi$ production in proton-proton collisions with PYTHIA8," Eur. Phys. J. C79 no. 1, (2019) 36, arXiv:1811.07744 [nucl-th].

[16] A. Ortiz, G. Bencedi, and H. Bello, "Revealing the source of the radial flow patterns in proton-proton collisions using hard probes," J. Phys. G44 no. 6, (2017) 065001, arXiv: 1608.04784 [hep-ph].

[17] ALICE Collaboration, S. Acharya et al., "Charged-particle production as a function of multiplicity and transverse spherocity in pp collisions at $\sqrt{s}=5.02$ and $13 \mathrm{TeV}$," arXiv: 1905.07208 [nucl-ex].

[18] ALICE Collaboration, J. Adam et al., "Enhanced production of multi-strange hadrons in high-multiplicity proton-proton collisions," Nature Phys. 13 (2017) 535-539, arXiv: 1606.07424 [nucl-ex].

[19] ALICE Collaboration, J. Adam et al., "Multi-strange baryon production in $\mathrm{p}-\mathrm{Pb}$ collisions at $\sqrt{s_{\mathbf{N N}}}=5.02$ TeV," Phys. Lett. B758 (2016) 389-401, arXiv:1512.07227 [nucl-ex]. 